\title{
Uncertainty and spatial updating in posterior parietal cortex ${ }^{1}$
}

\author{
Matteo Lisi ${ }^{2}$ \\ Department of Psychology, University of Essex, Colchester UK
}

Vision is an active process that requires frequently reorienting the central, high-acuity part of the retina toward relevant objects in the world to enable further processing. About three times per second we make saccadic eye movements that shift the entire image on the retina, yet the world appears stable and we can effortlessly keep track of where things are. The question of how the brain achieves visual stability across eye movements (or self-motion in general) has fascinated many great thinkers, including von Helmholtz (von Helmholtz, 1867) who had already recognized that our sense of location must come from a synthesis of retinal coordinates with information about eye position and movement. In recent years much progress has been made in uncovering the neural and computational mechanisms of visual stability. From a computational point of view, modeling studies have shown how populations of neurons could maintain a stable mapping between an internal retinotopic representation and spatiotopic (world-centered) coordinates of objects in the world (Casarotti et al., 2012; Denève et al., 2007; Zipser \& Andersen, 1988). These models rely either on proprioceptive signals from the eye to build an explicit spatiotopic map or on a copy of the motor command sent to move the eye, an efferent copy, that would be used to update the location of the target of interests on the internal retinotopic map. The latter mechanism can support a working spatiotopy that keeps track of locations across eye movements without postulating an explicit spatiotopic internal map. What concerns the neural mechanisms, a seminal study (Duhamel, Colby, et al., 1992) established a link between the processing of efferent copy signal and the posterior parietal cortex (PPC), by demonstrating the existence of neurons in the monkey lateral intraparietal area (LIP, which contains a retinotopic representation of the visual field) that, even before a saccade start, respond to the stimuli that will land in their receptive field after the eye movement. This result inspired investigations of visual stability in human patients with PPC damage (e.g. Duhamel, Goldberg, et al., 1992; Heide et al., 1995; Vuilleumier et al., 2007), leading to the conclusion that an intact parietal cortex is crucial for maintaining a stable sense of location across eye movements. A new study by Fabius and colleagues (Fabius et al., in press) however, present data that challenge this conclusion and call instead for a more nuanced view about the role of PPC in visual stability.

Many of the original studies on visual stability used an experimental protocol known as doublestep task. In this task, participants are asked to make two successive saccades toward the location of two sequentially flashed targets, both of which disappear before the first saccade begins. Thus,

\footnotetext{
${ }^{1}$ In press at Cortex, https://doi.org/10.1016/j.cortex.2020.02.013

2 Contact: $\underline{\text { m.lisi@essex.ac.uk }}$
} 
the correct execution of the second saccade, requires the memorized retinotopic location of the second target to be remapped in the post-saccadic coordinate system (i.e. relative to the new fixation point). Because patients with PPC lesion could only perform the second saccade correctly when the first saccade was directed towards the ipsilesional hemifield but not towards the contralesional one (Duhamel, Goldberg, et al., 1992; Heide et al., 1995), it was concluded that the deficit was in registering the amplitude and the direction of the contralesional first saccade. Although this clean-cut view has been very influential, later studies have put it into question. Rath-Wilson and Guitton (Rath-Wilson \& Guitton, 2015) tested PPC patients in two variations of the double-step task: First, they allowed patients to perform multiple saccades by giving them more time to complete the saccadic sequence. Second, to minimize the possibility that the failure in the double-step task was due to a general deficit in maintaining spatial location in memory, they presented the target in reverse order and also had a condition in which only the second target was presented and the choice of the first saccade amplitude was self-determined by the patient. Under these conditions, it was found that all patients tested were able to shift their gaze to the second target location, although they needed a larger number of shorter, hypometric saccades. In other words, when the task constraints are relaxed (by allowing more time and/or more saccades) the patients will eventually reach the correct location, implying that they have the necessary extra-retinal information available.

The new study by Fabius and colleagues (Fabius et al., in press) sheds intriguing new light on this issue. Fabius et al. designed a study to look at the perceptual aspects of remapping, using a protocol known as intrasaccadic displacement task. In brief, in this protocol participants make saccades to a peripheral target, which is then displaced slightly from its initial position while the saccade is still ongoing. Participants are then asked to report the direction of the displacement. Albeit small, such displacement would normally be highly visible if participants were not simultaneously moving their eyes. However, since it occurs during the saccade, the displacement becomes much less noticeable, likely because the visual system assumes that the world - and the saccade target - remain stable during eye movements (Mathôt \& Theeuwes, 2011). The interesting feature of this paradigm is that if the target is instead briefly extinguished, such that it reappears (on the displaced location) few hundreds of milliseconds later, participants' ability to detect and report the displacement improves significantly (Deubel et al., 1996). This so-called blanking effect is thought to reflect the increased use of extra-retinal information: since the target disappears, even if for a brief interval, the assumption of stability would be dropped, urging the visual system to use extra-retinal information for identifying what has changed. The rationale of the study is that if an intact parietal cortex is necessary to use extra-retinal signals for updating internal representation across saccades, then the blanking effect should be abolished in patients. Interestingly, the data revealed a more nuanced picture: patients showed a blanking effect, although the size of the effect was slightly smaller than that of healthy controls.

As Fabius et al. point out, these results convincingly show that the clean-cut conclusions of earlier studies need to be re-evaluated. Given more time, patients can complete the motor task, indicating that an intact PPC is not strictly necessary for a stable sense of location across eye 
movements. The issue of time constraints arises often in neuropsychological testing. For example, right-hemisphere damaged patients that would normally show signs of left visual peripersonal neglect in paper-and-pencil tests performed under strict time limits can complete the task correctly - thus not showing signs of neglect - when allowed more time (Priftis et al., 2019). Of course, requiring more time to complete the same task than healthy control participants should also be considered a deficit, but one of a different nature. Taken together, the studies by Fabius et al. and Rath-Wilson and Guitton (Fabius et al., in press; Rath-Wilson \& Guitton, 2015) show that although patients with PPC lesions can use extra-retinal information to maintain a stable sense of location across eye movements, they do not do so as efficiently as healthy participants, making both more variable and hypometric secondary saccades and more errors in their perceptual judgments.

In one recent study (Lisi et al., 2019) we reported some experimental results and a theory that could link together the perceptual and motor deficits in PPC patients. In our study, we hypothesized that normal saccade hypometria (about $10 \%$ of the required amplitude in healthy participants) could be better understood as a rational strategy for optimizing an asymmetrical cost function. The cost function is a measure of the relative desirability of each possible motor outcome, which would be used by the brain as a reference when deciding how to execute a movement. In our model the additional asymmetry assumption, simply put, would imply that whenever the oculomotor system is uncertain about the accurate amplitude that would land the gaze exactly on the target, it should prefer attempting short, rather than long, saccades ${ }^{3}$. This idea has several implications: for example that to minimize costs in the long run, the oculomotor system should produce shorter and more hypometric saccades when the uncertainty (about what would be the perfectly accurate saccade) is larger. Specifically, the model predicted a negative linear relationship between saccadic variability (standard deviation of saccadic amplitudes) and saccadic amplitude that was confirmed by the data. Furthermore, in separate conditions, we verified that the saccadic variability was also related to uncertainty in position judgments. Overall, our study revealed that increasing uncertainty yields both more variable perceptual judgments as well as slower and more hypometric saccades. Connecting the dots, our computational model of saccadic planning suggests that the deficit of PPC patients in the studies of Fabius and RathWilson (Fabius et al., in press; Rath-Wilson \& Guitton, 2015) could reflect a reduced precision in updating object locations across eye movements. According to this idea, PPC damage would either decrease the precision of extra-retinal information available, introduce noise in the computations underlying updating, or both. The increased uncertainty would, in the case of the double-step saccade task, yield second saccades that are more hypometric and slower, ultimately causing patients to use more saccades to reach the final location. In the intrasaccadic-displacement task, the larger uncertainty about the updated post-saccadic location of the target, would produce less accurate perceptual judgments. If the extra-retinal information is encoded by populations of

3 Although the cost function cannot be observed, in our study we found a way to indirectly test the assumption of asymmetry. We reasoned that saccades should try to avoid hypermetric errors because of the additional costs (latency) incurred when changing direction during a saccade sequence. Based on this we predicted that individuals with larger latency costs should have more asymmetrical cost functions, as revealed by the hypometria of their saccades, a prediction that found confirmation in the data. 
neurons (possibly redundantly across multiple maps in different areas of the brain), then lesioning a significant fraction of these neurons or maps may result in an overall noisier encoding of the extra-retinal signal, diminishing the ability of the brain to maintain a stable sense of location across eye movements but without preventing it completely. The idea that brain damage may reduce the precision of neural computation is not new: for example, patients with Parkinson's disease has been shown to display reduced precision in visual working memory tasks (Zokaei et al., 2014).

Assuming that PPC patients suffer from reduced precision in the efferent copy signal would piece together their motor and perceptual deficits. However, it is also possible that they have to rely on other mechanisms to maintain a stable sense of location across saccades, such as building a spatiotopic map using proprioceptive eye-position signals - a possibility that was flagged up also by Fabius et al. (Fabius et al., in press). Proprioceptive signals can arrive only after the eyes have moved, so this mechanism has been considered too slow to support visual stability. However, recent findings suggest that eye position signals are updated more rapidly than originally thought (Morris et al., 2012, 2013), and reach primary visual cortex with similar latency as retinal input (Morris \& Krekelberg, 2019). It is therefore conceivable that multiple redundant systems contribute to spatial updating of eye-movements, and which of these are affected in PPC patients remains to be elucidated.

Regardless of the precise mechanisms, the study by Fabius et al. provides a solid new demonstration that the involvement of PPC in maintaining a stable sense of location is more nuanced and less crucial than initially thought.

\section{References}

Casarotti, M., Lisi, M., Umiltà, C., \& Zorzi, M. (2012). Paying attention through eye movements: A computational investigation of the premotor theory of spatial attention. Journal of Cognitive Neuroscience, 24(7), 1519-31. https://doi.org/10.1162/jocn_a_00231

Denève, S., Duhamel, J.-R., \& Pouget, A. (2007). Optimal sensorimotor integration in recurrent cortical networks: A neural implementation of Kalman filters. The Journal of Neuroscience : The Official Journal of the Society for Neuroscience, 27(21), 5744-56. https://doi.org/10.1523/JNEUROSCI.398506.2007

Deubel, H., Schneider, W. X., \& Bridgeman, B. (1996). Postsaccadic target blanking prevents saccadic suppression of image displacement. Vision Research, 36(7), 985-996. https://doi.org/10.1016/00426989(95)00203-0

Duhamel, J. R., Colby, C. L., \& Goldberg, M. E. (1992). The updating of the representation of visual space in parietal cortex by intended eye movements. Science (New York, N.Y.), 255(5040), 90-2.

Duhamel, J. R., Goldberg, M. E., Fitzgibbon, E. J., Sirigu, A., \& Grafman, J. (1992). Saccadic dysmetria in a patient with a right frontoparietal lesion. The importance of corollary discharge for accurate spatial behaviour. Brain: A Journal of Neurology, 115 ( Pt 5), 1387-1402.

https://doi.org/10.1093/brain/115.5.1387 
Fabius, J. H., Nijboer, T. C. W., Fracasso, A., \& Van der Stigchel, S. (in press). Intra-saccadic displacement sensitivity after a lesion to the posterior parietal cortex. Cortex.

Heide, W., Blankenburg, M., Zimmermann, E., \& Kömpf, D. (1995). Cortical control of double-step saccades: Implications for spatial orientation: Double-Step Saccades and Cortex. Annals of Neurology, 38(5), 739748. https://doi.org/10.1002/ana.410380508

Lisi, M., Solomon, J. A., \& Morgan, M. J. (2019). Gain control of saccadic eye movements is probabilistic. Proceedings of the National Academy of Sciences, 116(32), 16137-16142. https://doi.org/10.1073/pnas.1901963116

Mathôt, S., \& Theeuwes, J. (2011). Visual attention and stability. Philosophical Transactions of the Royal Society of London. Series B, Biological Sciences, 366(1564), 516-27. https://doi.org/10.1098/rstb.2010.0187

Morris, A. P., Bremmer, F., \& Krekelberg, B. (2013). Eye-position signals in the dorsal visual system are accurate and precise on short timescales. The Journal of Neuroscience : The Official Journal of the Society for Neuroscience, 33(30), 12395-406. https://doi.org/10.1523/JNEUROSCI.0576-13.2013

Morris, A. P., \& Krekelberg, B. (2019). A Stable Visual World in Primate Primary Visual Cortex. Current Biology, 29(9), 1471-1480.e6. https://doi.org/10.1016/j.cub.2019.03.069

Morris, A. P., Kubischik, M., Hoffmann, K.-P., Krekelberg, B., \& Bremmer, F. (2012). Dynamics of eye-position signals in the dorsal visual system. Current Biology : CB, 22(3), 173-9. https://doi.org/10.1016/j.cub.2011.12.032

Priftis, K., Di Salvo, S., \& Zara, D. (2019). The importance of time limits in detecting signs of left visual peripersonal neglect: A multiple single-case, pilot study. Neurocase, 25(5), 209-215. https://doi.org/10.1080/13554794.2019.1658788

Rath-Wilson, K., \& Guitton, D. (2015). Refuting the hypothesis that a unilateral human parietal lesion abolishes saccade corollary discharge. Brain, 138(12), 3760-3775. https://doi.org/10.1093/brain/awv275

von Helmholtz, H. (1867). Handbuch der Physiologischen Optik. Voss.

Vuilleumier, P., Sergent, C., Schwartz, S., Valenza, N., Girardi, M., Husain, M., \& Driver, J. (2007). Impaired Perceptual Memory of Locations across Gaze-shifts in Patients with Unilateral Spatial Neglect. Journal of Cognitive Neuroscience, 19(8), 1388-1406. https://doi.org/10.1162/jocn.2007.19.8.1388

Zipser, D., \& Andersen, R. A. (1988). A back-propagation programmed network that simulates response properties of a subset of posterior parietal neurons. Nature, 331(6158), 679-84. https://doi.org/10.1038/331679a0

Zokaei, N., McNeill, A., Proukakis, C., Beavan, M., Jarman, P., Korlipara, P., Hughes, D., Mehta, A., Hu, M. T. M., Schapira, A. H. V., \& Husain, M. (2014). Visual short-term memory deficits associated with GBA mutation and Parkinson's disease. Brain, 137(8), 2303-2311. https://doi.org/10.1093/brain/awu143 